\title{
Search for an exosphere in sodium and calcium in the transmission spectrum of exoplanet 55 Cancri $e^{\star}$
}

\author{
A. R. Ridden-Harper ${ }^{1}$, I. A. G. Snellen ${ }^{1}$, C. U. Keller ${ }^{1}$, R. J. de Kok ${ }^{1}$, E. Di Gloria ${ }^{1}$, H. J. Hoeijmakers ${ }^{1}$, M. Brogi ${ }^{2}$,
} M. Fridlund ${ }^{1,3,4}$, B. L. A. Vermeersen ${ }^{5}$, and W. van Westrenen ${ }^{6}$

\author{
${ }^{1}$ Leiden Observatory, Leiden University, Niels Bohrweg 2, 2333 CA Leiden, The Netherlands \\ e-mail: arh@strw.leidenuniv.nl \\ 2 Center for Astrophysics and Space Astronomy, University of Colorado at Boulder, Boulder, CO 80309, USA \\ 3 Max-Planck Institute for Astronomy, Königstuhl 17, 69117 Heidelberg, Germany \\ ${ }^{4}$ Department of Earth and Space Sciences, Chalmers University of Technology, Onsala Space Observatory, 43992 Onsala, Sweden \\ 5 Delft University of Technology, Postbus 5, 2600 AA Delft, The Netherlands \\ ${ }^{6}$ Department of Earth Sciences, Vrije Universiteit Amsterdam, De Boelelaan 1085, 1081 HV Amsterdam, The Netherlands
}

Received 7 March 2016 / Accepted 16 June 2016

\begin{abstract}
Context. The atmospheric and surface characterization of rocky planets is a key goal of exoplanet science. Unfortunately, the measurements required for this are generally out of reach of present-day instrumentation. However, the planet Mercury in our own solar system exhibits a large exosphere composed of atomic species that have been ejected from the planetary surface by the process of sputtering. Since the hottest rocky exoplanets known so far are more than an order of magnitude closer to their parent star than Mercury is to the Sun, the sputtering process and the resulting exospheres could be orders of magnitude larger and potentially detectable using transmission spectroscopy, indirectly probing their surface compositions.

Aims. The aim of this work is to search for an absorption signal from exospheric sodium $(\mathrm{Na})$ and singly ionized calcium $\left(\mathrm{Ca}^{+}\right)$in the optical transmission spectrum of the hot rocky super-Earth 55 Cancri e. Although the current best-fitting models to the planet mass and radius require a possible atmospheric component, uncertainties in the radius exist, making it possible that 55 Cancri e could be a hot rocky planet without an atmosphere.

Methods. High resolution $(R \sim 110000)$ time-series spectra of five transits of 55 Cancri e, obtained with three different telescopes (UVES/VLT, HARPS/ESO $3.6 \mathrm{~m}$ and HARPS-N/TNG) were analysed. Targeting the sodium D lines and the calcium $\mathrm{H}$ and $\mathrm{K}$ lines, the potential planet exospheric signal was filtered out from the much stronger stellar and telluric signals, making use of the change of the radial component of the orbital velocity of the planet over the transit from -57 to $+57 \mathrm{~km} \mathrm{~s}^{-1}$.

Results. Combining all five transit data sets, we detect a signal potentially associated with sodium in the planet exosphere at a statistical significance level of $3 \sigma$. Combining the four HARPS transits that cover the calcium $\mathrm{H}$ and $\mathrm{K}$ lines, we also find a potential signal from ionized calcium $(4.1 \sigma)$. Interestingly, this latter signal originates from just one of the transit measurements - with a $4.9 \sigma$ detection at this epoch. Unfortunately, due to the low significance of the measured sodium signal and the potentially variable $\mathrm{Ca}^{+}$ signal, we estimate the $p$-values of these signals to be too high (corresponding to $<4 \sigma$ ) to claim unambiguous exospheric detections. By comparing the observed signals with artificial signals injected early in the analysis, the absorption by $\mathrm{Na}^{-} \mathrm{Ca}^{+}$are estimated to be at a level of $\sim 2.3 \times 10^{-3}$ and $\sim 7.0 \times 10^{-2}$ respectively, relative to the stellar spectrum.

Conclusions. If confirmed, the $3 \sigma$ signal would correspond to an optically thick sodium exosphere with a radius of $5 R_{\oplus}$, which is comparable to the Roche lobe radius of the planet. The $4.9 \sigma$ detection of $\mathrm{Ca}^{+}$in a single HARPS data set would correspond to an optically thick $\mathrm{Ca}^{+}$exosphere approximately five times larger than the Roche lobe radius. If this were a real detection, it would imply that the exosphere exhibits extreme variability. Although no formal detection has been made, we advocate that probing the exospheres of hot super-Earths in this way has great potential, also knowing that Mercury's exosphere varies significantly over time. It may be a fast route towards the first characterization of the surface properties of this enigmatic class of planets.
\end{abstract}

Key words. Planets and satellites: atmospheres - methods: data analysis - techniques: spectroscopic - planetary systems

\section{Introduction}

Transit and radial velocity surveys have revealed a new class of rocky planets orbiting their parent stars at very short distances

\footnotetext{
* Based on observations collected at the European Organisation for Astronomical Research in the Southern Hemisphere under ESO programmes 092.C-0178 and 288.C-5010 and the Telescopio Nazionale Galileo under programme CAT13B_33.
}

$\left(0.014-0.017 \mathrm{au}^{1}\right)$. Their evolutionary history is unknown. They may be rocky planets formed at significantly larger distances that subsequently migrated inwards, or could originally be gasrich planets which lost their gaseous envelopes during migration through tidal heating and/or direct stellar irradiation

\footnotetext{
10.014 is the semi-major axis of GJ $1214 \mathrm{~b}$, identified from exoplanets.org as the shortest semi-major axis with filter MINSI[mjupiter] $<10 M_{\oplus}$ and 0.017 comes from the definition of an ultra-short period
} planet (USP) from Demory et al. (2016b) of $P<0.75$ days. 
(Raymond et al. 2008). Insights into the composition of these hot rocky planets would help to distinguish between the different scenarios. Although the first secondary eclipse measurements have been presented in the literature (Demory et al. 2012), showing them to be indeed very hot, with observed surface or atmospheric temperatures of 1300-3000 K, detailed observations that could reveal atmospheric or surface compositions are beyond the reach of current instrumentation.

One physical process that could reveal information of a planet's surface composition, potentially also with current instruments, is that of sputtering. Atomic species are ejected from the planet surface by the intense stellar wind of charged particles, creating an extended exosphere around the planet. This process is well known from planet Mercury in our own solar system. It has an exosphere composed of atomic species including sodium $(\mathrm{Na})$, calcium $(\mathrm{Ca})$ and magnesium $(\mathrm{Mg})$, which are thought to be the results of sputtering, thermal vaporisation, photon-stimulated desorption, and meteoroid impact vaporisation. Since the discovery of an emission spectrum of sodium in the exosphere of Mercury by Potter \& Morgan (1985), it has been subsequently detected many times (see Killen et al. 2007, for a review) in emission, and less commonly in absorption during the transit of Mercury in front of the Sun (Potter et al. 2013). These decades of observations have revealed that sodium in the exosphere of Mercury shows a great deal of spatial and temporal variability. Rapid variations at a $50 \%$ level on timescales of a day in the ion-sputtering component of the sodium in Mercury's exosphere due to variability in the magnetosphere have been observed, as well as latitudinal and/or longitudinal variations (Potter \& Morgan 1990; Killen et al. 2007). In addition, long-term variations on timescales of months to years in photonstimulated desorption (Lammer et al. 2003; Killen et al. 2007) and radiation pressure acceleration (Smyth \& Marconi 1995; Killen et al. 2007) have been observed, as well as variations in meteoritic vaporisation (Morgan et al. 1988; Killen et al. 2007).

Mura et al. (2011) argued for the first time that such exospheres resulting from the sputtering process may be observable for hot rocky exoplanets. Their simulations for CoRoT-7b suggest that it may have a high escape rate of species such as $\mathrm{Na}$, $\mathrm{Ca}^{+}$, and $\mathrm{Mg}^{+}$which likely form a tail tens of planetary radii long. Guenther et al. (2011) observed a transit of CoRoT-7b with the UVES instrument on the VLT with a focus on $\mathrm{Na}, \mathrm{Ca}$, and $\mathrm{Ca}^{+}$. While Guenther et al. (2011) express their derived upper limits in units of stellar luminosity $\left(2-6 \times 10^{-6} L_{*}\right)$, these limits appear to correspond to an absorption level on the order of approximately $3 \times 10^{-3}$ smeared out over a $55 \mathrm{~km} \mathrm{~s}^{-1}$ velocity bin due to the change in the radial component of the orbital velocity of the planet during their long exposures. In this paper we target the exoplanet 55 Cancri e, whose host star has an apparent magnitude of $V=5.95,200$ times brighter than CoRoT-7.

In addition, Schaefer \& Fegley (2009) argue that a tidally locked hot rocky super-Earth could have a magma ocean that releases vapours to produce a silicate based atmosphere. Their models show that $\mathrm{Na}$ is likely the most abundant constituent of such an atmosphere, which they believe could form a large cloud of $\mathrm{Na}$ through interaction with the stellar wind.

Considerable progress regarding the detection and study of exospheres of hot gas giant exoplanets has already been made. Hydrogen exospheres extending beyond the Roche lobe have been repeatedly detected around HD 209458b (Vidal-Madjar et al. 2003, 2004) and HD 189733b (Lecavelier des Etangs et al. 2010), where the hydrogen signal from HD 189733b has been claimed to show temporal variation (Lecavelier des Etangs et al. 2012). Heavier atoms and ions have been detected in the exosphere of HD 209458b, including $\mathrm{C}^{+}$(Vidal-Madjar et al. 2004; Linsky et al. 2010) and, more tentatively, O (Vidal-Madjar et al. 2004), Mg (Vidal-Madjar et al. 2013) and $\mathrm{Si}^{2+}$ (Linsky et al. 2010). Exospheric studies have recently also been extended to smaller planets with the detection of hydrogen around the warm Neptune GJ 436b (Ehrenreich et al. 2015). We note that no hydrogen exosphere was detected around 55 Cancri e (Ehrenreich et al. 2012), which is the object of this study.

The hot, rocky super-Earth type planet, 55 Cancri e (or $\rho^{1}$ Cancri e, $55 \mathrm{Cnc}$ e) orbits a bright $(V=5.95) 0.905 M_{\odot}$ star. It has a very short orbital period of $17.7 \mathrm{~h}$ (see Table 1 for uncertainties), a radius of $2.173 R_{\oplus}$ (Gillon et al. 2012), a mass of $8.09 M_{\oplus}$, and an inferred average density of $5.51 \mathrm{~g} \mathrm{~cm}^{-3}$ (Nelson et al. 2014). Transits of $55 \mathrm{Cnc}$ e have been detected with broadband photometry from space in the visible (Winn et al. 2011) and infra-red (Demory et al. 2011), and recently also from the ground (de Mooij et al. 2014).

There is significant debate in the literature about the chemical composition and interior structure of $55 \mathrm{Cnc}$ e. Using the internal structure model by Valencia et al. (2006, 2010), Gillon et al. (2012) argue that $55 \mathrm{Cnc}$ e is likely a rocky, oxygenrich planet composed of silicates with a gaseous envelope consisting of either a mixture of hydrogen and helium of approximately $0.1 \%$ by mass or a water atmosphere of approximately $20 \%$ by mass. However, because such a low mass $\mathrm{H}-\mathrm{He}$ atmosphere would escape over a timescale of millions of years, while a water-vapour atmosphere could survive over billions of years, the water-vapour atmosphere interpretation is favoured, where the water-vapour is in a super-critical form due to its high temperature. Furthermore, Ehrenreich et al. (2012) found that $55 \mathrm{Cnc}$ e lacks a $\mathrm{H}$ exosphere which could be the result of complete $\mathrm{H}$ loss from the atmosphere in the past. In contrast, Madhusudhan et al. (2012) claim that if 55 Cnc e were to be a carbon-rich planet, a different structure is possible where $\mathrm{Fe}, \mathrm{C}$ (in the form of graphite and diamond), SiC, and silicates of a wide range of mass fractions could explain its density without the need for a gaseous envelope. While the $\mathrm{C} / \mathrm{O}$ ratio of $55 \mathrm{Cnc}$ was previously thought to be $>1$, a subsequent analysis by Teske et al. (2013) found that it more likely has a $\mathrm{C} / \mathrm{O}$ ratio of 0.8 . This value is lower than the value adopted by Madhusudhan et al. (2012) of $1.12 \pm 0.19$; however, it still corresponds to the predicted minimum value of 0.8 necessary for the formation of a carbon-rich condensate under the assumption of equilibrium (Larimer 1975).

Furthermore, Demory et al. (2016b) report a $4 \sigma$ detection of variability in the day-side thermal emission of $55 \mathrm{Cnc}$ e, with the emissions varying by a factor of 3.7 between 2012 to 2013 . They also tentatively suggest variations in the transit depth and calculate the planetary radii to range from $1.75 \pm 0.13 R_{\oplus}$ to $2.25 \pm 0.17 R_{\oplus}$ with a mean value of $1.92 \pm 0.08 R_{\oplus}$, which is approximately $2 \sigma$ smaller than the value published by Gillon et al. (2012) of $2.17 \pm 0.10 R_{\oplus}$ based on Spitzer + MOST data. We believe that this smaller radius implies that the need for a significant atmosphere to explain the planet's radius is significantly reduced.

If $55 \mathrm{Cnc}$ e does not have an atmosphere, its surface would be directly exposed to stellar radiation, making it analogous to Mercury. It is likely that the processes which produce the exosphere of Mercury would be much more pronounced on $55 \mathrm{Cnc} e$ because it receives a bolometric flux from its host star that is approximately 500 times greater than Mercury receives from the Sun. This corresponds to an equilibrium temperature of $55 \mathrm{Cnc} e$ of almost 2000 K. Demory et al. (2016b) claim to have detected 
Table 1. Properties of 55 Cancri e.

\begin{tabular}{lcc}
\hline \hline Parameter & Value & Source \\
\hline \multicolumn{1}{c}{ Stellar properties } & & \\
& & \\
Distance $(\mathrm{pc})$ & $12.34 \pm 0.11$ & van Leeuwen $(2007)$ \\
Radius $\left(R_{\odot}\right)$ & $0.943 \pm 0.010$ & von Braun et al. $(2011)$ \\
Luminosity $\left(L_{\odot}\right)$ & $0.582 \pm 0.014$ & von Braun et al. $(2011)$ \\
$T_{\mathrm{EFF}}(\mathrm{K})$ & $5196 \pm 24$ & von Braun et al. $(2011)$ \\
Mass $\left(M_{\odot}\right)$ & $0.905 \pm 0.015$ & von Braun et al. $(2011)$ \\
$\log g$ & $4.45 \pm 0.01$ & von Braun et al. $(2011)$ \\
Radial velocity & $27.58 \pm 0.07$ & Nidever et al. $(2002)$ \\
& &
\end{tabular}

Planet properties

\begin{tabular}{lcc} 
Period (days) & $0.7365449 \pm 0.000005$ & Gillon et al. (2012) \\
Orb. radius (AU) & $0.0154 \pm 0.0001$ & $\dagger^{\dagger}$ \\
Radius $\left(R_{\oplus}\right)$ & $2.173 \pm 0.098$ & Gillon et al. (2012) \\
Mass $\left(M_{\oplus}\right)$ & $8.09 \pm 0.26$ & Nelson et al. (2014) \\
Density $\left(\mathrm{g} \mathrm{cm}^{-3}\right)$ & $5.51 \pm \pm_{1.00}^{1.32}$ & Nelson et al. (2014) \\
\hline
\end{tabular}

Notes. ${ }^{(\dagger)}$ Calculated using Kepler’s third law.

brightness temperatures which vary from $1300 \mathrm{~K}$ to $3000 \mathrm{~K}$; however, the mechanism which causes this variability is not understood.

Demory et al. (2016a) report the observation of a complete phase curve of $55 \mathrm{Cnc}$ e in the $4.5 \mu \mathrm{m}$ channel of the Spitzer Space Telescope Infrared Array Camera which allowed them to construct a longitudinal thermal brightness map due to $55 \mathrm{Cnc}$ e being tidally locked to its host star. This map revealed that $55 \mathrm{Cnc}$ e has a strong day-night temperature contrast with temperatures of $2700 \mathrm{~K}$ and $1380 \mathrm{~K}$ on the day and night sides respectively. Furthermore, they found that the day side exhibits highly asymmetric thermal emissions, with a hot spot located $41 \mathrm{deg}$ east of the substellar point. These observations were interpreted as being either due to an atmosphere with heat recirculation confined to the day side only, or a planet without an atmosphere with low-viscosity magma flows on the surface. Atmospheric escape rate arguments indicate that it is unlikely that $55 \mathrm{Cnc}$ e has a thick atmosphere, so the magma ocean interpretation is favoured.

In this paper, we aim to search for an absorption signal from exospheric sodium $(\mathrm{Na})$ and singly ionized calcium $\left(\mathrm{Ca}^{+}\right)$in the optical transmission spectrum of the hot rocky super-Earth $55 \mathrm{Cnc}$ e. This paper is structured as follows. Section 2 describes the data and Sect. 3 explains the methods used in this analysis. Section 4 presents the results, and Sect. 5 discusses the interpretation of the results and concludes.

\section{Observational data}

High-dispersion spectral time series of five transits of $55 \mathrm{Cnc}$ e taken with three different telescopes were used for our analysis. The five data sets each cover one transit including observations just before and after the transit. We observed one transit using the Ultraviolet and Visual Echelle Spectrograph (UVES; Dekker et al. 2000) installed on the Nasmyth B focus of the Very Large Telescope (VLT) at the Paranal Observatory. Furthermore, we retrieved additional data sets from observatory archives. Two of these were observed with the High Accuracy Radial Velocity Planet Searcher (HARPS; Mayor et al. 2003) located at the ESO
$3.6 \mathrm{~m}$ Telescope at the La Silla Observatory, and two from its northern-hemisphere copy - HARPS-N (Cosentino et al. 2012) located at the $3.6 \mathrm{~m}$ Telescopio Nazionale Galileo at the Roque de los Muchachos Observatory. An overview of all observations is shown in Table 4.

\subsection{UVES data}

138 UVES spectra were obtained of 55 Cnc. The transit timing, dates, exposure times, observational cadence and phase coverage are presented in Table 4 . The observations were made using the red arm of UVES, utilizing grating CD\#3 with a central wavelength of $580.0 \mathrm{~nm}$, resulting in a wavelength range of 4726.5-6835.1 $\AA$. A resolving power of $R \approx 110000$ was achieved using a slit width of $0.3^{\prime \prime}$ and image slicer \#3 to minimize the slit losses. Using no charge-coupled device (CCD) binning, the sampling is two pixels per spectral element (D'Odorico et al. 2000).

Unfortunately, cirrus clouds were present during our observations which considerably decreased the signal-to-noise ratio $(\mathrm{S} / \mathrm{N})$ in the spectra, ranging from $S / N=180$ during relatively good spells down to $S / N=60$ per pixel.

\subsection{HARPS data}

The HARPS data used for our analysis cover two transits and were originally taken for ESO programme 288.C-5010 (PI: A. Triaud) which was used by López-Morales et al. (2014) to investigate the Rossiter-Mclaughlin effect in $55 \mathrm{Cnc}$ e. We retrieved the pipeline-reduced data from the ESO Science Archive Facility $^{2}$.

HARPS has a resolving power of $R \approx 115000$ and a wavelength range of 3800-6910 $\mathrm{A}$. It is enclosed in a vacuum vessel, pressure and temperature controlled to a precision of $\pm 0.01 \mathrm{mbar}$ and $\pm 0.01 \mathrm{~K}$ respectively, resulting in a wavelength precision of $\lesssim 0.5 \mathrm{~m} \mathrm{~s}^{-1}$ night $^{-1}$ (Bonfils et al. 2013). It has two fibres which feed the spectrograph with light from the telescope and calibration lamp. The fibre aperture on the sky is $1^{\prime \prime}$. The CCD has a pixel size of $15 \mu \mathrm{m}$ and a sampling of 3.2 pixels per spectral element (Mayor et al. 2003). The transit timing, dates, exposure times, observational cadence, and phase coverage are presented in Table 4.

\subsection{HARPS-N data}

The HARPS-N observations also cover two transits, and were originally taken in TNG Observing programme CAT13B_33 (PI: F. Rodler), also to investigate the Rossiter McLaughlin effect by the same team (López-Morales et al. 2014). The pipelinereduced data was retrieved by us from the TNG data archive ${ }^{3}$.

HARPS-N is a copy of HARPS so its properties are all identical or very similar to HARPS at ESO. It has a slightly different wavelength range of 3830-6900 $\AA$ and a sampling of 3.3 pixels per FWHM. It also has a greater temperature stability than HARPS of $0.001 \mathrm{~K}$, giving a short-term precision of $0.3 \mathrm{~m} \mathrm{~s}^{-1}$ and a global long-term precision of better than $0.6 \mathrm{~m}^{-14}$. The fibre aperture on sky and spectral resolution are identical to those of HARPS. The transit timing, dates, exposure times, observational cadence, and phase coverage are presented in Table 4.

\footnotetext{
2 http://archive.eso.org/cms.html

http://ia2 .oats.inaf.it/archives/tng

http://www.tng.iac.es/instruments/harps/
} 
We note that an additional five publicly available data sets $^{5}$ of the transit of 55 Cnc e were obtained with HARPS$\mathrm{N}$ by Bourrier \& Hébrard (2014) to investigate the RossiterMcLaughlin effect. The individual spectra of these data sets have exposure times of $360 \mathrm{~s}$, which is twice the average exposure time of the data used in this study. Therefore, we chose to not use these data sets because due to the very rapid change in the radial component of the orbital velocity of the planet $\left(114 \mathrm{~km} \mathrm{~s}^{-1}\right.$ over the transit), any planet signature would be smeared out by ten pixels, significantly decreasing its peak.

\section{Data analysis}

In our analysis we concentrate on the $\mathrm{H}$ and $\mathrm{K}$ lines of ionized calcium (at $3968.47 \AA$ and $3933.66 \AA$ respectively) and the two sodium D lines (5889.95 $\AA$ and $5895.92 \AA$ ). While the sodium lines are covered by all data sets, the ionized calcium lines are only present in the HARPS and HARPS-N data and not in the UVES data.

\subsection{Processing of UVES spectra}

The observed spectra are dominated by stellar and possible telluric absorption lines which are orders of magnitude stronger than the expected planet features. Since the stellar and telluric absorption lines are quasi-fixed in wavelength (the stellar lines change in radial velocity by approximately $1.4 \mathrm{~m} \mathrm{~s}^{-1}$ during the four hour observations McArthur et al. 2004) and the radial component of the orbital velocity of the planet changes by tens of $\mathrm{km} \mathrm{s}^{-1}$ during the transit, the change in the Doppler shift of the planet lines can be used to separate the planet signal from that of the star and the Earth's atmosphere. The procedure we used to carry out this processing is very similar to that used in previous work (eg. Snellen et al. 2010; Hoeijmakers et al. 2015) and the individual steps are summarised below.

1. Extraction of wavelength calibrated 1D spectra. The UVES data were reduced using the standard ESO UVES reduction pipeline (Ballester et al. 2000) which was executed with Gasgano ${ }^{6}$ and EsoRex ${ }^{7}$. The pipeline produced a onedimensional wavelength calibrated spectrum for each order for each exposure.

2. Normalization of the spectra to a common flux level. Variation in instrumental throughput (for example, due to slit losses) and atmospheric absorption result in the spectra having different baseline fluxes. To normalise the individual spectra to a common flux level, every spectrum was divided through its median value. The median value of a spectrum was used to minimize the influence of cosmic ray hits. This scaling can be performed because this analysis does not depend on the absolute flux, but instead only on the relative changes in flux as a function of wavelength.

3. Alignment of spectra. It is important for our analysis that all of the individual stellar spectra are in the same intrinsic wavelength frame. Since the radial component of the barycentric velocity changes during an observing night, and the absolute wavelength solution of UVES is unstable at the subpixel level, the spectra need to be re-aligned to a common wavelength frame. To do this, Gaussians were fitted to

\footnotetext{
5 TNG programme IDs: OPT12B_13,OPT13B_30, OPT14A_34

6 https://www.eso.org/sci/software/gasgano.html

http: //www . eso.org/sci/software/cpl/esorex.html
}

narrow stellar lines close to the sodium D lines in each spectrum to determine the offset relative to a Kurucz model stellar spectrum with atmospheric parameters of $T_{\text {eff }}=5000 \mathrm{~K}$, $\log (g)=4.5$ (Castelli \& Kurucz 2004) that was Doppler shifted to account for the system velocity of $55 \mathrm{Cnc}$ of $27.58 \pm 0.07 \mathrm{~km} \mathrm{~s}^{-1}$. These offsets were then used to update the intrinsic wavelength solution for the star. The normalized and aligned spectra are shown in the top panel of Fig. 1.

4. Removal of cosmic rays. The standard UVES data reduction recipes do not remove cosmic ray hits for observations made with the image slicer. Therefore, after the UVES spectra were normalized and aligned, cosmic rays were removed by fitting a linear function at each wavelength position through all spectra, so that cosmic rays could be identified as being outliers from the fit. They were then replaced with the interpolated value from the fit. This process was iterated twice with different threshold values so it only identified very strong cosmic rays on the first iteration. This was necessary because the presence of very strong cosmic rays could skew the linear fit and cause weaker cosmic rays to be missed.

5. Removal of the stellar absorption features. The stellar spectrum of $55 \mathrm{Cnc}$ was assumed to be constant during a night of observations. This allowed the stellar features to be removed by dividing every observed spectrum from a single night by the median of all of the observed spectra from that night. This only slightly weakened the strength of potential planet absorption lines because they changed wavelength significantly ( $>100$ pixels) during the transit. The resulting spectra are shown in the second panel of Fig. 1.

6. Removal of large systematic trends. Significant systematic trends in the residual spectra in the wavelength direction became apparent after the stellar features had been removed. These trends, which differed for different spectra, were fitted with a linear slope, that was subsequently removed at the beginning of Step 2 (see above). Steps 2 to 5 were redone, after which we proceeded with Step 7.

7. Removal of telluric lines with principal component analysis (PCA). Telluric absorption lines change in strength, mainly due to the change in airmass during observations, but also possibly due to variations in the water vapour content of the Earth's atmosphere. We removed the telluric absorptions in the sodium D region using PCA (also know as singular value decomposition) over the time domain. This method relies on the assumption that all of the telluric lines vary in the same way and is discussed in Sect. 2 of de Kok et al. (2013). Since Step 3 of our data analysis aligned the spectra to the stellar rest frame, the telluric lines show a small shift in position during the night since they are in the rest frame of the observer. However, the PCA algorithm was able to mostly remove the misaligned telluric lines, as shown in the third panel of Fig. 1, while the components that were removed are shown in Fig. 2. Some weak residual features from the telluric lines remain after the PCA. These are probably caused by the line width of the telluric lines changing slightly during the night. The PCA algorithm is a blind process so if it is allowed to remove a large number of components, it will eventually remove all variation in the data, including the planet signal. However, only four PCA components were required to remove the telluric lines. By injecting artificial planet signals (see Sect. 3.4 and the lower panel of Fig. 1) we show that the planet signal is left intact by this procedure.

8. Weighting by noise as a function of wavelength. The values at each wavelength position were subsequently weighted down by the noise, derived from the standard deviation of 
A. R. Ridden-Harper et al.: Search for an exosphere in sodium and calcium in the transmission spectrum
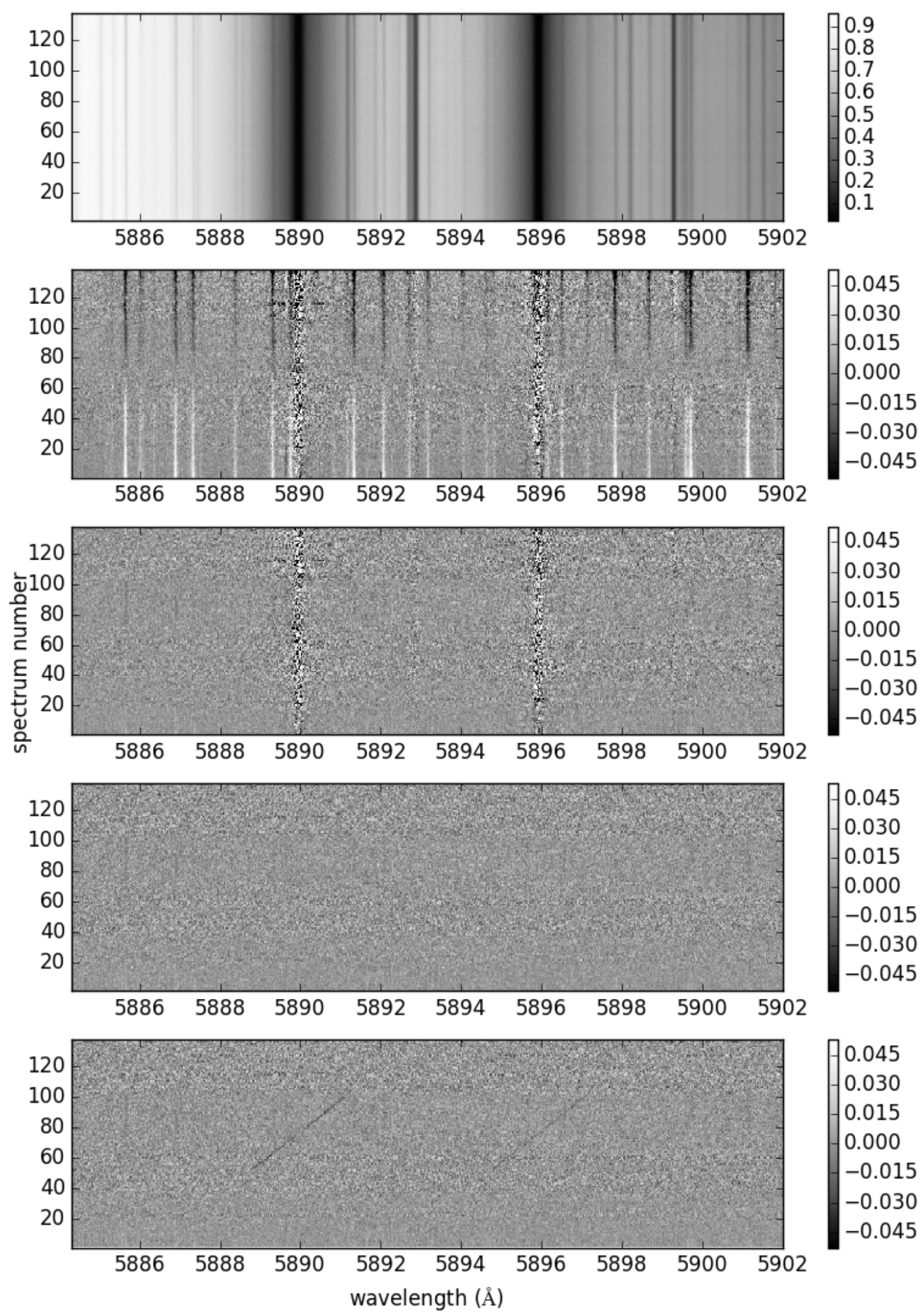

Fig. 1. Visual representation of the processing steps as described in Sect. 3. The vertical axis of each matrix represents the sequence number of the observed spectrum. This figure shows the UVES data, but the HARPS and HARPS-N datasets look very similar. The first panel shows the data around the sodium D lines after normalization and alignment in Steps 2 and 3. The second panel shows the residual matrix after dividing through the average star spectrum (Step 5). The third panel shows the residuals after the PCA analysis (Step 7), and the fourth panel shows the same after normalizing each column of the matrix by its standard deviation (Step 8). The bottom panel shows the same data, but after injecting an artificial planet signal before Step 3 - at a level of $3 \%$ of the stellar flux. The injected planet signal can be seen as a diagonal trace from spectrum number 53 to 99 , resulting from the change in the radial component of the planet orbital velocity during transit. 


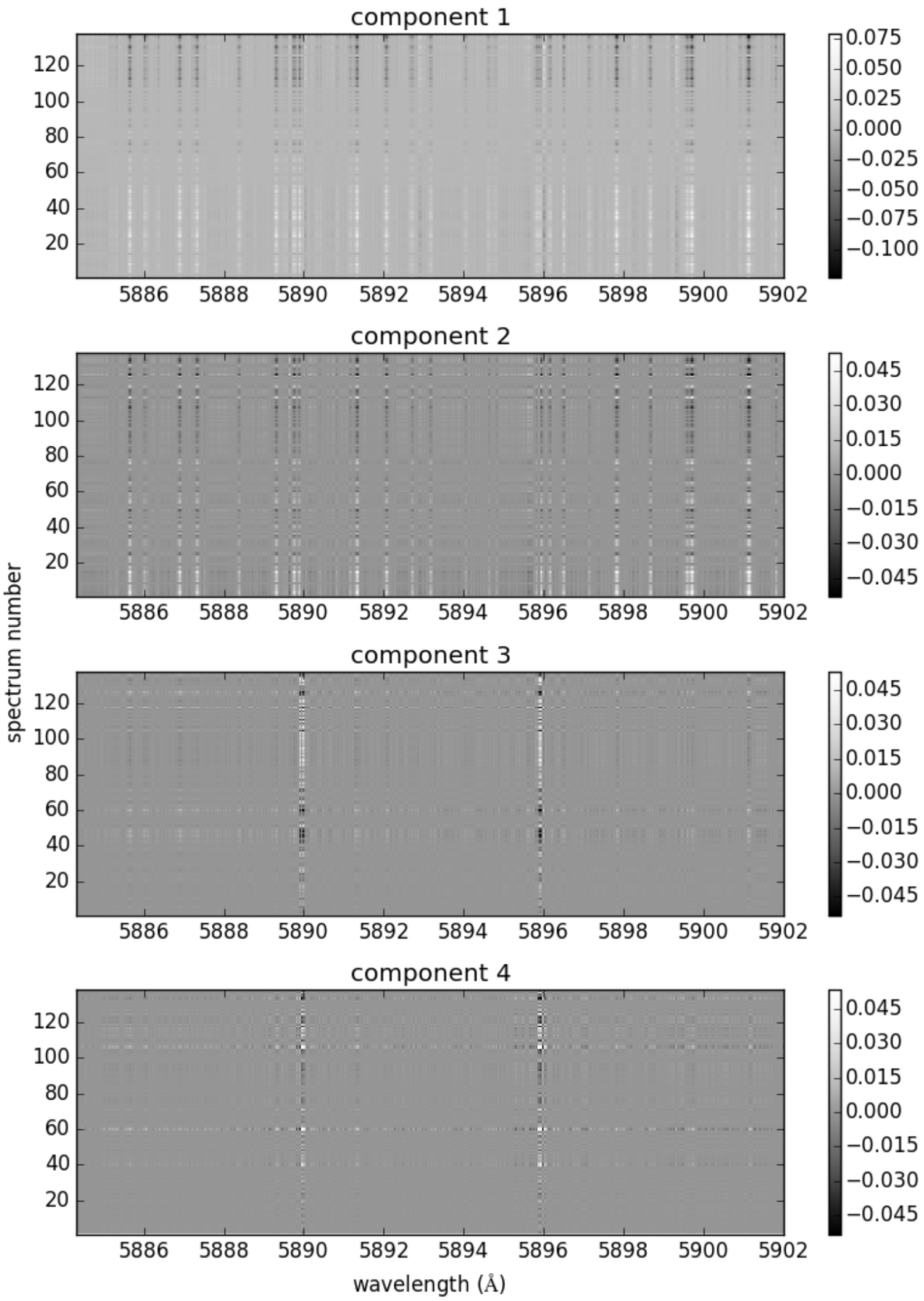

Fig. 2. Components that were removed by the principle component analysis (PCA) algorithm to remove telluric lines around the Na D lines in the UVES data set. The top panel shows the first component and subsequent panels show the subsequent components, until the fourth component in the bottom panel. The top panel has a different colour scale to the other panels.

the residuals as function of time at that position. Practically, this only influences the region directly around the cores of the two stellar sodium D lines, as can be seen by comparing panels 3 and 4 of Fig. 1. This naturally weighs down the contribution from the spectra during which the planet absorption overlaps with the strong stellar sodium lines. The effect this has on the planet signal is illustrated in panel 5 of Fig. 1. An artificial planet signal is injected into the observed 
Table 2. Signal-to-noise-ratios $(\mathrm{S} / \mathrm{N})$ of the data sets.

\begin{tabular}{lccc}
\hline \hline Dataset & $\begin{array}{c}\text { Average } S / N \\
\text { per spectrum }\end{array}$ & $\begin{array}{c}\text { Number of spectra } \\
\text { during transit }\end{array}$ & Total $S / N$ \\
\hline UVES & 116.3 & 47 & 977 \\
HARPS-N B & 161.2 & 18 & 838 \\
HARPS-N A & 223.3 & 19 & 1192 \\
HARPS B & 109.6 & 24 & 658 \\
HARPS A & 143.9 & 24 & 863 \\
\hline
\end{tabular}

spectra (see Sect. 3.4) as a $3 \%$ absorption relative to the stellar spectrum. This signal is weighted with a box-shaped transit profile which is reasonable for a small planet such as $55 \mathrm{Cnc}$ e. At the mid-transit point when the planet signal is at the same wavelength as the stellar signal (and thus falls in the cores of the Na D lines), the retrieved planet signal is reduced by a factor of approximately 10 .

\subsection{Processing of HARPS and HARPS-N data}

Except for small differences, the processing of the HARPS and HARPS-N data was performed in a similar way to that of the UVES data explained above. Since the data retrieved from the data archives of both telescopes is completely reduced and wavelength-calibrated, Step 1 was not needed. In addition, the wavelength calibration of both HARPS and HARPS-N is stable at the $1 \mathrm{~m} \mathrm{~s}^{-1}$ level, and delivered to the user in the restframe of the star. Therefore also step 3 was not needed.

In addition to the sodium D lines (5889.95 $\AA$ and $5895.92 \AA$ ), the HARPS and HARPS-N spectra also cover the $\mathrm{Ca} H$ and K lines (at $3968.47 \AA$ and $3933.66 \AA$ respectively). While the sodium lines are covered by all data sets, the ionized calcium $\mathrm{H}$ and $\mathrm{K}$ lines are only present in the HARPS and HARPS-N data and not in the UVES data.

\subsection{Combining the different data sets}

The final step in the analysis is to merge the signal from the two sodium D lines (and calcium $\mathrm{H}$ and $\mathrm{K}$ lines) and combine the signal from all the in-transit spectra. In addition, we also combine the data sets from the different telescopes.

Two regions of $16 \AA$ centred on the positions of the $\mathrm{Na} \mathrm{D}_{2}$ and $\mathrm{D}_{1}$ lines in the residual spectra were averaged with weights proportional to the relative theoretical line strengths. Each spectrum was subsequently shifted to the planet rest frame and added in time over the transit. The signal from the different data sets was subsequently combined using weights proportional to the total in-transit $\mathrm{S} / \mathrm{N}$ of the data set. The unfavourable observing conditions during the UVES observations caused the UVES observations to have a comparable total $\mathrm{S} / \mathrm{N}$ to the HARPS and HARPS-N observations (see Table 2).

The final data from the calcium $\mathrm{H}$ and $\mathrm{K}$ lines were produced in the same way using a weighting ratio of $\mathrm{Ca} \mathrm{K} / \mathrm{Ca} H=2$ for the two lines.

\subsection{Injection of artificial planet signals}

A useful technique to determine the magnitude of the absorption signal of the planet relative to the stellar spectrum is to inject artificial planet signals early on in the data processing so that the artificial signals are processed in the same way as a real signal would be. This also allows us to check to what level our
Table 3. An estimation of the width of the absorption signal from $55 \mathrm{Cnc}$ e based on the average change of its radial velocity during the exposure of each spectrum in each data set.

\begin{tabular}{lccc}
\hline \hline Dataset & Width $\left(\mathrm{km} \mathrm{s}^{-1}\right)$ & Width $(\AA)$ & Width (pixels) \\
\hline UVES & 1.3 & 0.026 & 1.0 \\
HARPS A & 4.0 & 0.078 & 4.9 \\
HARPS B & 4.0 & 0.078 & 4.9 \\
HARPS-N A & 5.3 & 0.105 & 6.5 \\
HARPS-N B & 5.3 & 0.105 & 6.5 \\
\hline
\end{tabular}

analysis removes any planet signal. The injection of artificial signals was carried out in a similar way to Snellen et al. (2010) and Hoeijmakers et al. (2015).

The artificial planet signals of the sodium $D_{1}(5895.92 \AA)$ and $\mathrm{D}_{2}(5889.95 \AA)$ lines were generated using Gaussian line profiles of equal width, and with amplitudes with a ratio of $D_{2} / D_{1}=2$. These relative line strengths were calculated according to Eq. (1) in Sharp \& Burrows (2007). We do note that these equations in principle only hold for local thermodynamic equilibrium, while planet exospheres may be better described by radiative transfer algorithms which use a non-Maxwellian velocity distribution function such as in Chaufray \& Leblanc (2013). The quantum parameters which describe the $\mathrm{Na} \mathrm{D}$ line transitions were obtained from the Vienna Atomic Line Database (VALD; Kupka et al. 2000). The relative strengths of the $\mathrm{Na} D$ lines are practically independent of temperature across the range of 1000 to $3000 \mathrm{~K}$. We assumed $T=2000 \mathrm{~K}$.

Using the orbital parameters from Gillon et al. (2012) and assuming a circular orbit (Demory et al. 2012), the radial velocity of the planet can be calculated at the time of each exposure to determine the central wavelengths of the Doppler-shifted sodium lines.

The planet signal was injected into the in-transit data by multiplying the observed spectra with the artificial absorption model according to

$F(\lambda)_{\text {injected }}=\left[1-A \times F_{\text {model }}\left(\lambda, v_{\text {rad }}\right)\right] F_{\text {obs }}(\lambda)$,

where $F_{\text {obs }}(\lambda)$ is the observed spectrum, $F_{\text {model }}\left(\lambda, v_{\text {rad }}\right)$ is the Doppler shifted sodium model spectrum, with $A$ as a scaling parameter that sets the amplitude of the sodium $D_{2}$ line, and $F(\lambda)_{\text {injected }}$ is the output spectrum.

During an exposure, the radial component of the orbital velocity of the planet changes significantly. For example, the observations taken with HARPS-N have an exposure time of $240 \mathrm{~s}$, during which the planet radial velocity changes by approximately $5 \mathrm{~km} \mathrm{~s}^{-1}$, corresponding to six resolution elements. Thus, even for an intrinsically narrow planet absorption, the observed signal cannot be narrower than five or six pixels. Therefore, the injected artificial sodium lines have a width equal to this observational broadening, which is different for each data set, as shown in Table 3.

The same procedure was used to inject an artificial absorption signal of ionized calcium, using a relative line ratio of $\mathrm{Ca} \mathrm{K} / \mathrm{Ca} H=2$ as calculated from Sharp \& Burrows (2007).

\section{Results}

\subsection{Sodium}

The results for sodium are shown in Fig. 3. The left and right panels show the unbinned data and data binned by five pixels (or $0.05 \AA$ ) respectively. From top to bottom the panels show the two 
Table 4. Observational timing parameters.

\begin{tabular}{|c|c|c|c|c|c|}
\hline $\begin{array}{l}\text { data set } \\
\text { Program nr. }\end{array}$ & $\begin{array}{l}\text { UVES } \\
\text { ESO: 092.C-0178 }\end{array}$ & $\begin{array}{l}\text { HARPS-N B } \\
\text { TNG: CAT13B_33 }\end{array}$ & $\begin{array}{l}\text { HARPS-N A } \\
\text { TNG: CAT13B_33 }\end{array}$ & $\begin{array}{l}\text { HARPS B } \\
\text { ESO: } 288 . C-5010\end{array}$ & $\begin{array}{l}\text { HARPS A } \\
\text { ESO: 288.C-5010 }\end{array}$ \\
\hline \multirow{5}{*}{$\begin{array}{l}\text { date (UTC) } \\
\text { start } \\
\text { phase } \\
\text { end } \\
\text { phase } \\
\text { cadence (s) } \\
\text { exposure } \\
\text { time (s) }\end{array}$} & 2014-01-04 & 2013-11-29 & 2013-11-15 & $2012-03-16$ & $2012-01-28$ \\
\hline & 0.871 & 0.850 & 0.850 & 0.944 & 0.939 \\
\hline & 0.106 & 0.108 & 0.074 & 0.077 & 0.093 \\
\hline & 109.0 & 265.8 & 264.3 & 211.4 & 211.4 \\
\hline & 60 & 240 & 240 & 180 & $180^{\dagger}$ \\
\hline \multirow{5}{*}{$\begin{array}{l}\text { observation } \\
\text { start (UTC) } \\
\text { transit } \\
\text { start (UTC) } \\
\text { mid-transit } \\
\text { time (UTC) } \\
\text { transit } \\
\text { end (UTC) } \\
\text { observation } \\
\text { end (UTC) }\end{array}$} & 04:43:03.805 & 02:10:18.576 & 02:18:54.502 & 01:01:25.593 & 03:56:16.478 \\
\hline & $06: 16: 30.913$ & $04: 05: 54.424$ & 04:14:02.316 & $01: 17: 26.886$ & $04: 16: 50.727$ \\
\hline & 07:00:13.153 & 04:49:36.664 & $04: 57: 44.556$ & 02:01:09.126 & 05:00:32.967 \\
\hline & 07:43:55.393 & 05:33:18.904 & $05: 41: 26.797$ & 02:44:51.366 & 05:44:15.207 \\
\hline & $08: 52: 37.259$ & $06: 44: 16.990$ & $06: 15: 53.853$ & $03: 22: 45.983$ & 06:39:14.017 \\
\hline \multirow{4}{*}{$\begin{array}{l}\text { Nr. spectra } \\
\text { pre-transit } \\
\text { Nr. spectra } \\
\text { in transit } \\
\text { Nr. spectra } \\
\text { post-transit } \\
\text { total nr. } \\
\text { of spectra }\end{array}$} & 52 & 26 & 25 & 5 & 6 \\
\hline & 47 & 18 & 19 & 24 & 24 \\
\hline & 39 & 17 & 9 & 12 & 17 \\
\hline & 138 & 61 & 53 & 41 & 47 \\
\hline
\end{tabular}

Notes. The orbital phases of $55 \mathrm{Cnc}$ e are based on the orbital parameters derived from the Spitzer + MOST observations used in Gillon et al. (2012). ${ }^{(\dagger)}$ Except for the first two spectra which have exposure times of $123 \mathrm{~s}$.

HARPS data sets, the two from HARPS-N, the UVES data set, and the signal combined from all telescopes. In these panels, the stellar and telluric signals have been removed so all that remains is residual noise and a possible absorption signal from $55 \mathrm{Cnc}$ e. The noise has an approximately Gaussian distribution, so the statistical significance of the detection can be estimated by comparing the depth of the absorption signal to the standard deviation of the noise. Since any planet signal is expected to be broadened due to the long exposure times, the $\mathrm{S} / \mathrm{N}$ in the unbinned data, calculated as described above, may be underestimated.

While there is a hint of planet absorption in the individual UVES data set, this is somewhat more pronounced in the combined (binned) data. This signal has a statistical significance of $3.2 \sigma$ and $3.3 \sigma$ in the unbinned and binned data respectively. The binned and unbinned versions of the combined data are also overlayed in Fig. 4 for clarity.

By injecting artificial signals at various levels relative to the stellar spectrum we can estimate the strength of the retrieved signal. If real, the planet sodium lines in the combined data are at a level of $2.3 \times 10^{-3}$ with respect to the star.

\subsection{Ionized calcium}

The results for ionized calcium are shown in Fig. 5. The individual panels are the same as in Fig. 3, except that UVES is not included because the wavelength range of the UVES data does not cover the calcium $\mathrm{H}$ and $\mathrm{K}$ lines. In contrast to the sodium data, an interesting signal can be seen in the first HARPS-A data set. It shows a feature that has a statistical significance of $4.9 \sigma$, although it is blueshifted with respect to the planet rest frame by approximately $4 \mathrm{~km} \mathrm{~s}^{-1}$. An overlay of the binned and unbinned data of the HARPS-A data set is shown in Fig. 6. The signal does not appear in the other data sets, resulting in a S/N of less than 4 in the combined data.
The contribution to the $4.9 \sigma \mathrm{Ca}^{+}$signal from each individual spectrum of the HARPS-A data set is shown in Fig. 7. This figure presents the data in the rest-frame of $55 \mathrm{Cnc}$ e so that the features that contribute to the signal lie on a vertical line that is blueshifted by approximately $4 \mathrm{~km} \mathrm{~s}^{-1}$. The transit duration is indicated in the figure. It can be seen that there are contributions from multiple spectra during transit, indicating that the signal is not caused by a random spurious feature in a single spectrum. If the exosphere is extended beyond the Roche lobe, one would expect it to be distorted and hence possess different velocities relative to the planet and possibly be detectable just before or after transit. However, the $\mathrm{S} / \mathrm{N}$ in the data is not sufficient to see such distortions or extended absorption signatures.

If real, the planet calcium $\mathrm{H}$ and $\mathrm{K}$ lines in the HARPS-A data set are at a level of $7.0 \times 10^{-2}$ with respect to the star.

To assess whether the $\mathrm{Ca}^{+}$signal could originate from variability in the stellar $\mathrm{Ca}^{+} \mathrm{H}$ and $\mathrm{K}$ lines, we investigated the emission from the cores of the $\mathrm{H}$ and $\mathrm{K}$ lines in all data sets (Fig. 8). We found no evidence for variations in excess of that expected from Poisson noise within each transit data set. Although one dataset (HARPS-N A, hence not corresponding to that showing $\mathrm{Ca}^{+}$) exhibits stronger $\mathrm{Ca} \mathrm{H}+\mathrm{K}$ emission (55 Cnc has a known 39 day periodicity in its $\mathrm{Ca}^{+} \mathrm{H}$ and $\mathrm{K}$ stellar emission lines; Fischer et al. 2008), it shows no variability during the transit. Also, the radial velocity of $55 \mathrm{Cnc}$ e changes by $\pm 57 \mathrm{~km} \mathrm{~s}^{-1}$ which causes a Doppler shift of $\pm 0.75 \AA$ relative to the core of the lines. Since the signals across spectra are combined in the planet rest frame, only the spectra taken close to the midtransit point (where the planet signal is at the same wavelength as the stellar lines) could be influenced by variability in the $\mathrm{Ca}^{+}$ $\mathrm{H}$ and $\mathrm{K}$ emission. Therefore, even if there was some variability in emission during a night of observations, its impact on the results would still be limited. 

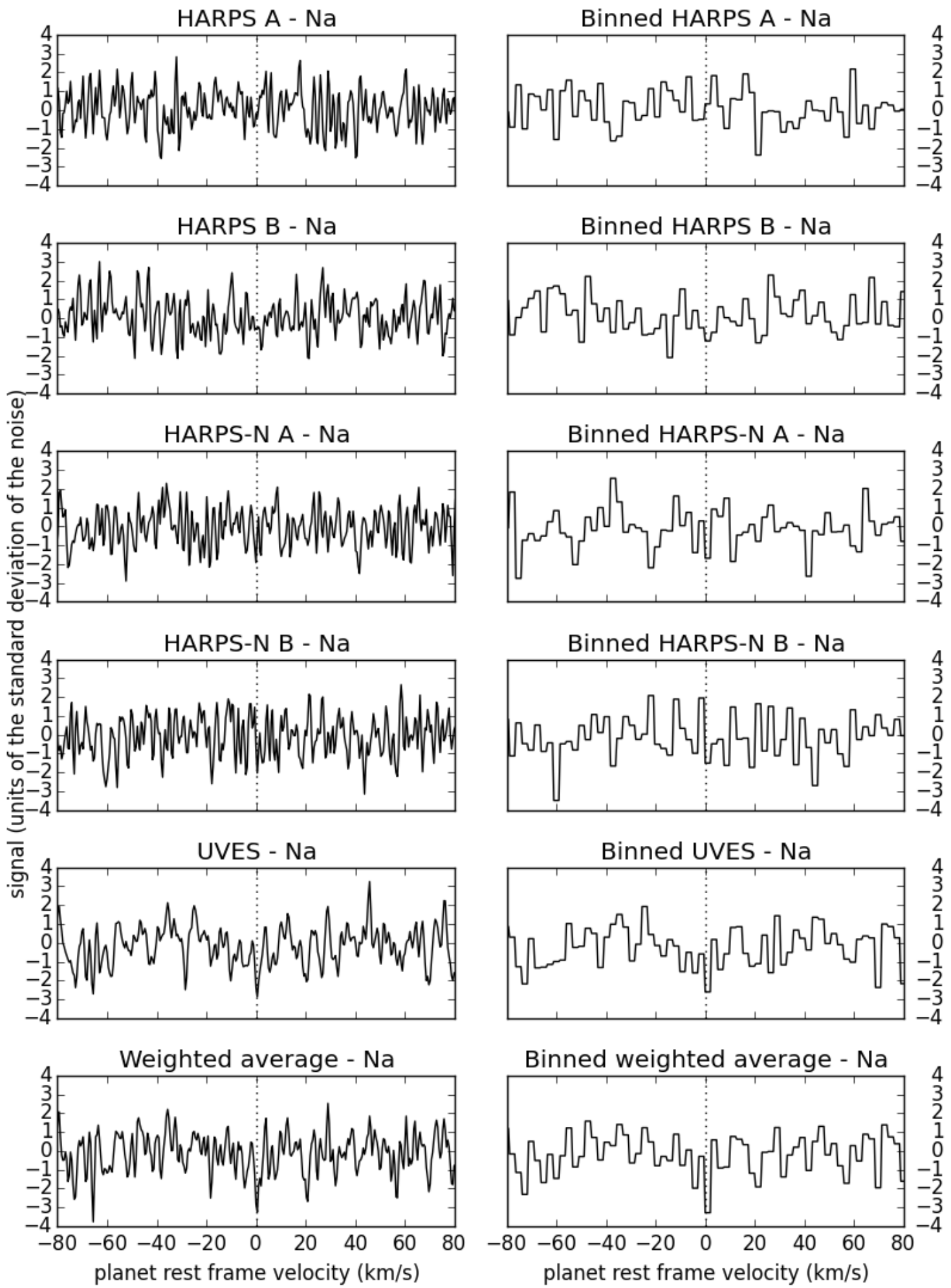

Binned weighted average - $\mathrm{Na}$

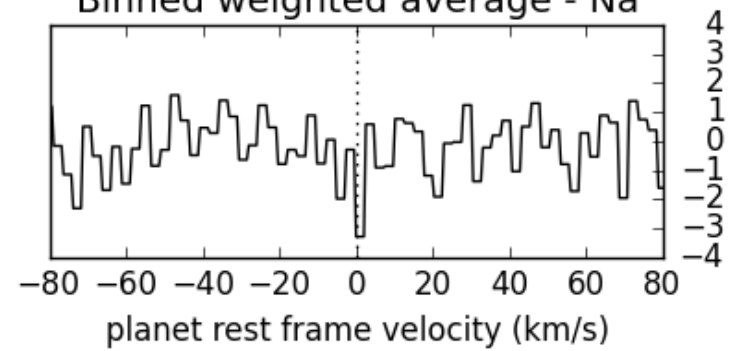

Fig. 3. Signal of sodium from the individual data sets and from an average of the data sets, weighted according to the $\mathrm{S} / \mathrm{N}$ of the data set. The signals have been scaled to be in units of the standard deviation of the noise. The left column is not binned while the right column is binned every $0.05 \AA$ or $3.8 \mathrm{~km} \mathrm{~s}^{-1}$. The binned average signal has a detection $S / N=3.3$. The vertical dotted line in all panels indicates a planet rest frame velocity of $0 \mathrm{~km} \mathrm{~s}^{-1}$.

\section{Discussion and conclusions}

In this paper we carried out a search for neutral sodium $(\mathrm{Na})$ and singly ionized calcium $\left(\mathrm{Ca}^{+}\right)$in the exosphere of the exoplanet $55 \mathrm{Cnc}$ e with transmission spectroscopy. This search yielded a
$3.3 \sigma$ detection of $\mathrm{Na}$ after combining five transit data sets and a $4.9 \sigma$ detection of $\mathrm{Ca}^{+}$in only one transit data set.

We estimated the $p$-value of the $\mathrm{Ca}^{+}$detection in one of our four HARPS(-N) data sets. The probability of observing a 


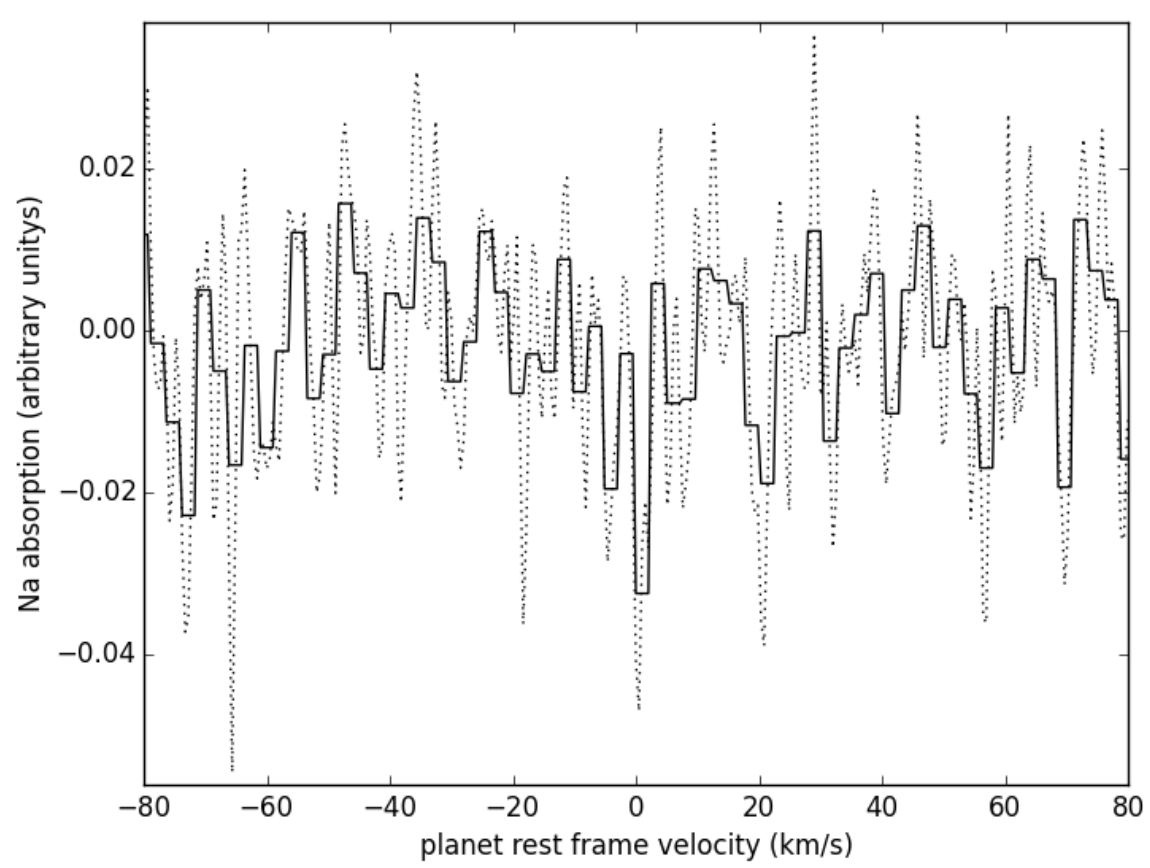

Fig. 4. Average signal of sodium from all data sets both not binned (dotted line) and binned (solid black line) every $0.05 \AA$ or $3.8 \mathrm{~km} \mathrm{~s}{ }^{-1}$ (as shown in the bottom panels of Fig. 3). This binning regime results in a detection that has a $\mathrm{S} / \mathrm{N}$ of approximately $3.3 \sigma$.

spurious $4.9 \sigma$ signal is very low at $4.8 \times 10^{-7}$. However, we would have observed such a signal at any velocity between -50 and $+50 \mathrm{~km} \mathrm{~s}^{-1}$ in the planet rest frame, corresponding to about approximately 20 positions. Multiplying this by the number of transits observed means that we had approximately 80 opportunities to observe a spurious signal, meaning that we can estimate that the false alarm probability is $\sim 80 \times 4.8 \times 10^{-7} \approx 4 \times 10^{-5}$, corresponding to $<4 \sigma$. For this estimate we do not take into account our freedom to use a certain width for the probed signal. In addition, the possible impact of unquantified correlated noise in the data may also increase the $p$-values. We therefore think these data are as yet insufficient to claim definite detections of the planet exosphere.

As discussed above, the spectral resolution of any potential planet signal is broadened due to the change of the radial component of the planet orbital velocity during an exposure. As shown in Table 3, this "instrumental" broadening is five to six pixels for the HARPS and HARPS-N data, and below the intrinsic spectral resolution of the spectrograph for the UVES data, due to the significantly shorter exposure times. In addition, the absorption from sodium and ionized calcium could be intrinsically broadened due to a strong velocity field in the planet exosphere (Mura et al. 2011). If the observed signals from either sodium or ionized calcium are real, they are indeed broad at the five to six pixel $\left(4 \mathrm{~km} \mathrm{~s}^{-1}\right)$ level, which is much broader than the intrinsic width of the $\mathrm{Na}_{2}$ line in the exosphere of Mercury, previously observed by Potter et al. (2013), of approximately $20 \mathrm{m \AA}$ or approximately $1 \mathrm{~km} \mathrm{~s}^{-1}$.

It is not clear whether to expect the blueshift of approximately $4 \mathrm{~km} \mathrm{~s}^{-1}$ as measured for the potential ionized calcium signal. On the one hand, the photon-ionization lifetime of singly-ionized calcium is estimated to be significantly longer than that of neutral sodium, which could allow it to accumulate a significant acceleration as it is picked up by the stellar wind and dragged in the anti-stellar direction. On the other hand, if the planet has a significant dipole magnetic field, the ionized calcium may be trapped in the planet's magnetic field (Mura et al. 2011).

If the ionized calcium signal is real, it would imply that this signal is highly variable, since it is only visible in one of the four data sets. As discussed in the introduction, we do know that Mercury's exosphere is highly variable, on a range of timescales from days, to months, to years (Killen et al. 2007). It is not clear at this stage whether we would expect similar behaviour for the exospheres of hot rocky super-Earths. If $55 \mathrm{Cnc}$ e were to have a significant atmosphere, a confirmed detection would be evidence of atmospheric blow-off. On the other hand, if $55 \mathrm{Cnc}$ e does not have a thick atmosphere, as is suggested by Demory et al. (2016a) as being a likely interpretation of its longitudinal thermal brightness map, the exosphere would likely be produced by sputtering of the surface.

We modelled the exosphere of $55 \mathrm{Cnc}$ e to first order as an optically thick ring around the planet. Ignoring subtle effects like stellar limb darkening, the fraction of starlight absorbed by sodium and ionized calcium in the exosphere of $55 \mathrm{Cnc}$ e would correspond to an outer radius of the exosphere of $5 R_{\oplus}$ and $25 R_{\oplus}$ respectively -2.3 and 12 times the radius of the planet. We compare this to the Roche radius of $55 \mathrm{Cnc}$ e, calculated using

$R_{R}=\frac{0.49 q^{2 / 3}}{0.6 q^{2 / 3}+\ln \left(1+q^{1 / 3}\right)}$,

where $q=M_{\text {planet }} / M_{\text {star }}$ (Eggleton 1983), which is found to be $R_{R}=5.35 R_{\oplus}$. Hence, the possible sodium signal, if optically thick, would come from a region as large as the planet's Roche lobe, while that of ionized calcium would be significantly larger. If the sodium exosphere were not optically thick, it would also need to be significantly larger than the planet's Roche lobe. If the $\mathrm{Ca}^{+}$exosphere really were to have a radius of $25 R_{\oplus}$, it would have an earlier ingress and a delayed egress compared to what would be expected from the radius of the planet as determined by broadband photometry. Using Mandel \& Agol (2002), the transit duration for $55 \mathrm{Cnc}$ e with a $25 R_{\oplus}$ exosphere was found to last 26 min longer than the broadband transit duration. This corresponds to a range of orbital phases of -0.054 to 0.054 . There 
A. R. Ridden-Harper et al.: Search for an exosphere in sodium and calcium in the transmission spectrum
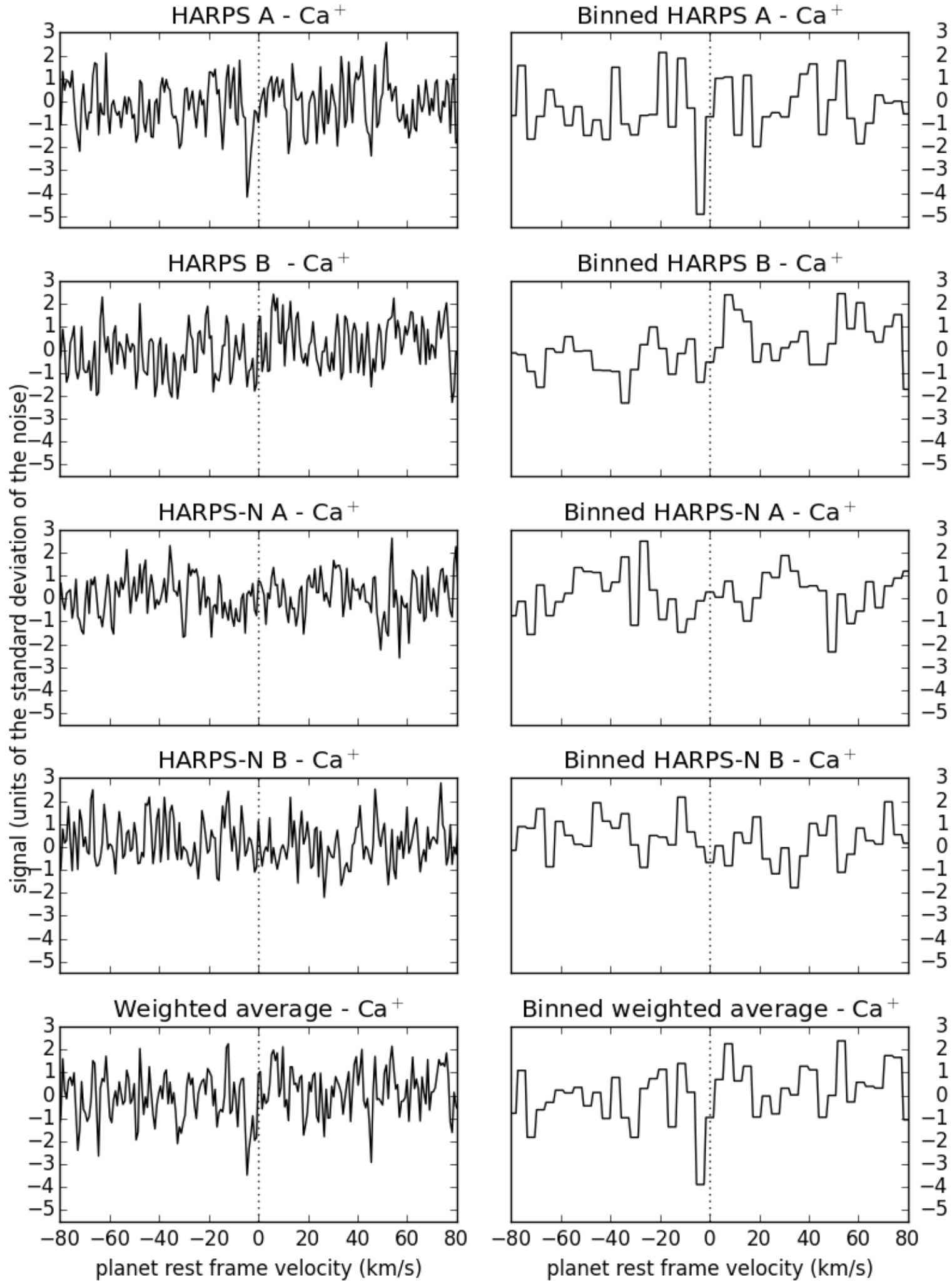

Fig. 5. Signal of ionized calcium from the individual data sets and from an average of the data sets, weighted according to the $\mathrm{S} / \mathrm{N}$ of the data set. The signals have been scaled to be in units of the standard deviation of the noise. The left column is not binned while the right column is binned every $0.05 \AA$ or $3.8 \mathrm{~km} \mathrm{~s}^{-1}$. The vertical dotted line in all panels indicates a planet rest frame velocity of $0 \mathrm{~km} \mathrm{~s}^{-1}$. The binned average signal has a detection $S / N=4.1$; however, this completely originates from the HARPS A data set which individually has a binned detection $\mathrm{S} / \mathrm{N}$ of 4.9 .

may be a hint of this early ingress and delayed egress, as can be seen in Fig. 7, however, the $\mathrm{S} / \mathrm{N}$ is not sufficient to make definite claims.
Although no formal detection has been made, we advocate that probing the exospheres of hot super-Earths in this way has great potential, also knowing that Mercury's exosphere varies 


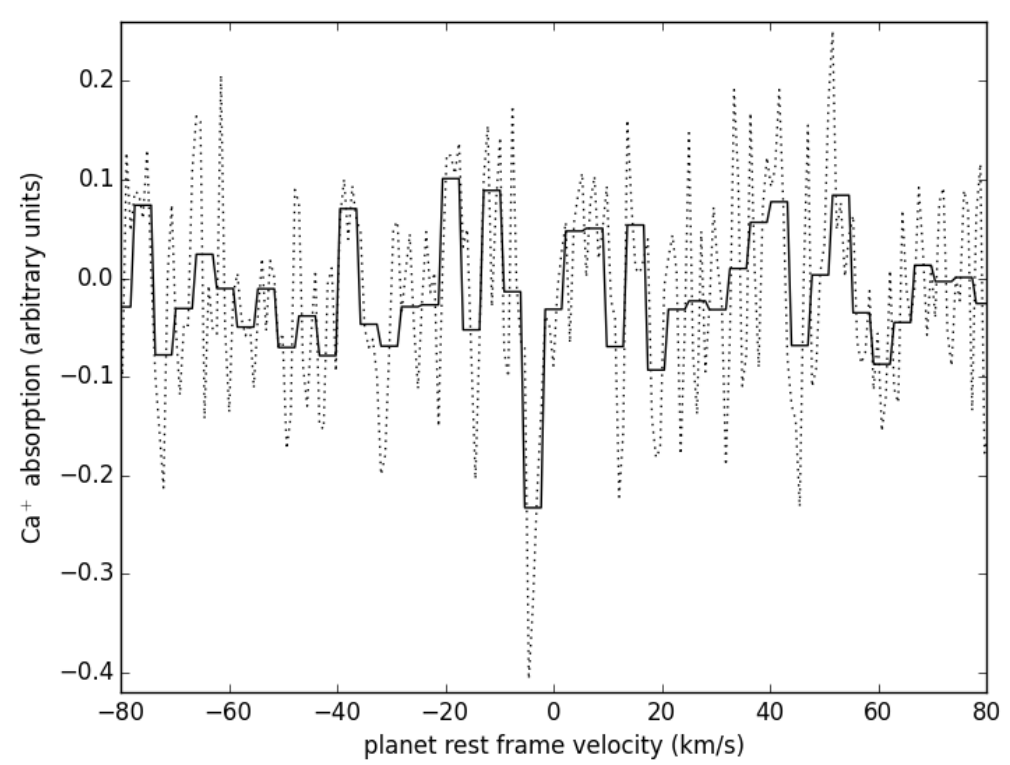

Fig. 6. Signal of ionized calcium from the HARPS A data set both not binned (dotted line) and binned (solid black line) every $0.05 \AA$ or $3.8 \mathrm{~km} \mathrm{~s}{ }^{-1}$ (as shown in the bottom panels of Fig. 5). This binning regime results in a detection that has a $\mathrm{S} / \mathrm{N}$ of approximately $4.9 \sigma$.

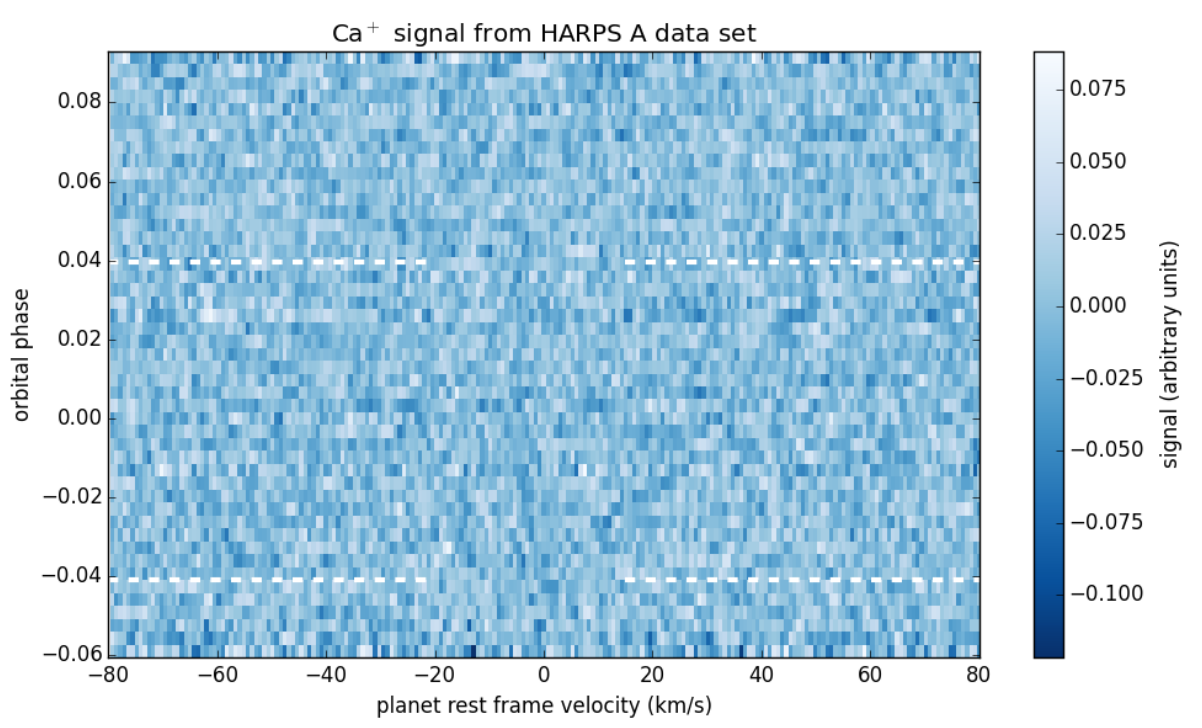

Fig. 7. Trace of the signal of $\mathrm{Ca}^{+}$from the HARPS A data set across the time-series of spectra in the rest frame of $55 \mathrm{Cnc}$ e. In this frame, the planet signal lies on a vertical line, blueshifted by approximately $4 \mathrm{~km} \mathrm{~s}^{-1}$. Dashed horizontal white lines indicate the transit duration.
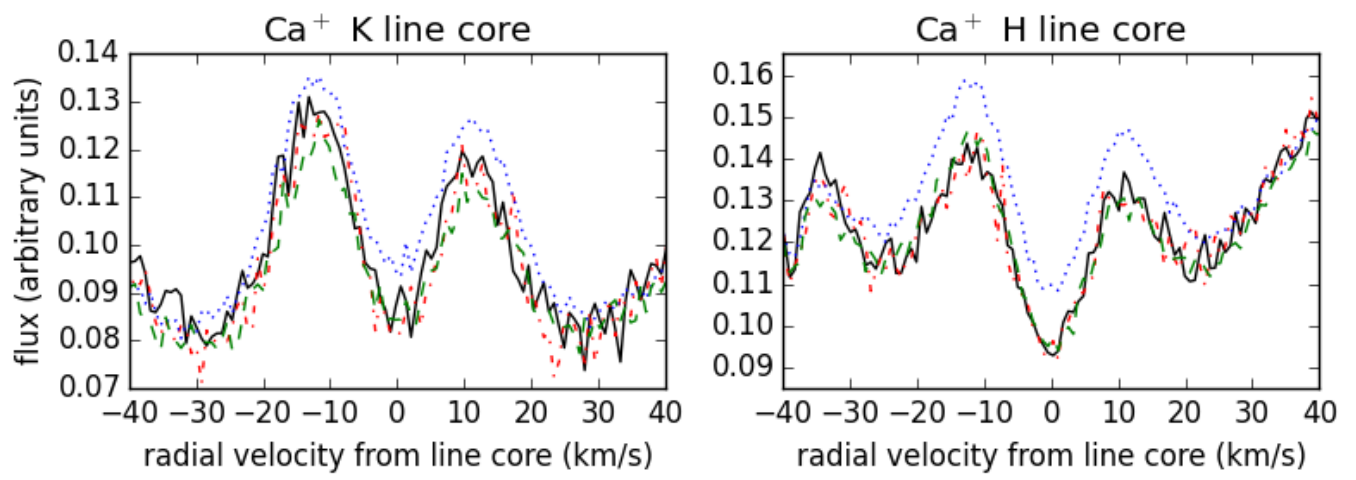

Fig. 8. Median spectrum of each data set showing the median emission from the cores of the $\mathrm{Ca}^{+} \mathrm{H}($ right $)$ and $\mathrm{K}$ (left) lines. The solid black line is the HARPS A data set, the dotted-dashed red line is the HARPS B data set, the dotted blue line is the HARPS-N A data set and the dashed green line is the HARPS-N B data set. The two panels have different vertical scales. 
significantly over time. It may be a fast route towards the first characterization of the surface properties of this enigmatic class of planets. Our team is pursuing a transit monitoring programme with UVES to further investigate the possible variable signal from ionized calcium.

Acknowledgements. A.R.R.-H. is grateful to the Planetary and Exoplanetary Science (PEPSci) programme of the Netherlands Organisation for Scientific Research (NWO) for support. I.A.G.S. acknowledges support from an NWO VICI grant (639.043.107). M.B. acknowledges support by NASA, through Hubble Fellowship grant HST-HF2-51336 awarded by the Space Telescope Science Institute. We thank the anonymous referee for their constructive comments.

\section{References}

Ballester, P., Modigliani, A., Boitquin, O., et al. 2000, The Messenger, 101, 31 Bonfils, X., Delfosse, X., Udry, S., et al. 2013, A\&A, 549, A109 Bourrier, V., \& Hébrard, G. 2014, A\&A, 569, A65

Castelli, F., \& Kurucz, R. L. 2004, ArXiv e-prents [arXiv: astro-ph/0405087]

Chaufray, J.-Y., \& Leblanc, F. 2013, Icarus, 223, 975

Cosentino, R., Lovis, C., Pepe, F., et al. 2012, in SPIE Conf. Ser., 8446, 1

de Kok, R. J., Brogi, M., Snellen, I. A. G., et al. 2013, A\&A, 554, A82

de Mooij, E. J. W., López-Morales, M., Karjalainen, R., Hrudkova, M., \& Jayawardhana, R. 2014, ApJ, 797, L21

Dekker, H., D’Odorico, S., Kaufer, A., Delabre, B., \& Kotzlowski, H. 2000, in Series, 4008, Optical and IR Telescope Instrumentation and Detectors, eds. M. Iye, \& A. F. Moorwood, SPIE Conf. 534

Demory, B.-O., Gillon, M., Deming, D., et al. 2011, A\&A, 533, A114

Demory, B.-O., Gillon, M., Seager, S., et al. 2012, ApJ, 751, L28

Demory, B.-O., Gillon, M., de Wit, J., et al. 2016a, Nature, 532, 207

Demory, B.-O., Gillon, M., Madhusudhan, N., \& Queloz, D. 2016b, MNRAS, 455,2018

D’Odorico, S., Cristiani, S., Dekker, H., et al. 2000, in Discoveries and Research Prospects from 8- to 10-Meter-Class Telescopes, ed. J. Bergeron, SPIE Conf. Ser., 4005, 121

Eggleton, P. P. 1983, ApJ, 268, 368

Ehrenreich, D., Bourrier, V., Bonfils, X., et al. 2012, A\&A, 547, A18

Ehrenreich, D., Bourrier, V., Wheatley, P. J., et al. 2015, Nature, 522, 459

Fischer, D. A., Marcy, G. W., Butler, R. P., et al. 2008, ApJ, 675, 790

Gillon, M., Demory, B.-O., Benneke, B., et al. 2012, A\&A, 539, A28
Guenther, E. W., Cabrera, J., Erikson, A., et al. 2011, A\&A, 525, A24

Hoeijmakers, H. J., de Kok, R. J., Snellen, I. A. G., et al. 2015, A\&A, 575, A20

Killen, R., Cremonese, G., Lammer, H., et al. 2007, Space Sci. Rev., 132, 433

Kupka, F., Ryabchikova, T., Piskunov, N., Stempels, H., \& Weiss, W. 2000, Balt. Astron., 9, 590

Lammer, H., Wurz, P., Patel, M. R., et al. 2003, Icarus, 166, 238

Larimer, J. W. 1975, Geochim. Cosmochim. Acta, 39, 389

Lecavelier des Etangs, A., Ehrenreich, D., Vidal-Madjar, A., et al. 2010, A\&A, 514, A72

Lecavelier des Etangs, A., Bourrier, V., Wheatley, P. J., et al. 2012, A\&A, 543, L4

Linsky, J. L., Yang, H., France, K., et al. 2010, ApJ, 717, 1291

López-Morales, M., Triaud, A. H. M. J., Rodler, F., et al. 2014, ApJ, 792, L31

Madhusudhan, N., Lee, K. K. M., \& Mousis, O. 2012, ApJ, 759, L40

Mandel, K., \& Agol, E. 2002, ApJ, 580, L171

Mayor, M., Pepe, F., Queloz, D., et al. 2003, The ESO Messenger, 114, 20

McArthur, B. E., Endl, M., Cochran, W. D., et al. 2004, ApJ, 614, L81

Morgan, T. H., Zook, H. A., \& Potter, A. E. 1988, Icarus, 75, 156

Mura, A., Wurz, P., Schneider, J., et al. 2011, Icarus, 211, 1

Nelson, B. E., Ford, E. B., Wright, J. T., et al. 2014, MNRAS, 441, 442

Nidever, D. L., Marcy, G. W., Butler, R. P., Fischer, D. A., \& Vogt, S. S. 2002, ApJS, 141, 503

Potter, A., \& Morgan, T. 1985, Science, 229, 651

Potter, A. E., \& Morgan, T. H. 1990, Science, 248, 835

Potter, A. E., Killen, R. M., Reardon, K. P., \& Bida, T. A. 2013, Icarus, 226, 172

Raymond, S. N., Barnes, R., \& Mandell, A. M. 2008, MNRAS, 384, 663

Schaefer, L., \& Fegley, B. 2009, ApJ, 703, L113

Sharp, C. M., \& Burrows, A. 2007, ApJS, 168, 140

Smyth, W. H., \& Marconi, M. L. 1995, ApJ, 441, 839

Snellen, I. A. G., de Kok, R. J., de Mooij, E. J. W., \& Albrecht, S. 2010, Nature, 465, 1049

Teske, J. K., Cunha, K., Schuler, S. C., Griffith, C. A., \& Smith, V. V. 2013, ApJ, 778,132

Valencia, D., O’Connell, R. J., \& Sasselov, D. 2006, Icarus, 181, 545

Valencia, D., Ikoma, M., Guillot, T., \& Nettelmann, N. 2010, A\&A, 516, A20

van Leeuwen, F. 2007, A\&A, 474, 653

Vidal-Madjar, A., Lecavelier des Etangs, A., Désert, J.-M., et al. 2003, Nature, 422, 143

Vidal-Madjar, A., Désert, J.-M., Lecavelier des Etangs, A., et al. 2004, ApJ, 604, L69

Vidal-Madjar, A., Huitson, C. M., Bourrier, V., et al. 2013, A\&A, 560, A54

von Braun, K., Boyajian, T. S., ten Brummelaar, T. A., et al. 2011, ApJ, 740, 49

Winn, J. N., Matthews, J. M., Dawson, R. I., et al. 2011, ApJ, 737, L18 